Check for updates

Cite this: RSC Adv., 2018, 8, 39066

Received 4th September 2018

Accepted 15th November 2018

DOI: $10.1039 / c 8 \mathrm{ra07379a}$

rsc.li/rsc-advances

\section{Studies on structure-function relationships of acetolactate decarboxylase from Enterobacter cloacae}

\author{
Fangling Ji, (D) $\dagger^{* a}$ Yanbin Feng, $\dagger^{\mathrm{b}}$ Mingyang $\mathrm{Li}^{\mathrm{a}}{ }^{\mathrm{a}}$ Yongliang Yang, ${ }^{\mathrm{a}}$ Tianqi Wang, ${ }^{\mathrm{a}}$ \\ Jingyun Wang, (D) ${ }^{a}$ Yongming Bao ${ }^{\text {ac }}$ and Song Xue (iD *b
}

Acetoin is an important bio-based platform chemical with wide applications. Among all bacterial strains, Enterobacter cloacae is a well-known acetoin producer via $\alpha$-acetolactate decarboxylase (ALDC), which converts $\alpha$-acetolactate into acetoin and is identified as the key enzyme in the biosynthetic pathway of acetoin. In this work, the enzyme properties of Enterobacter cloacae ALDC (E.C.-ALDC) were characterized, revealing a $K_{\mathrm{m}}$ value of $12.19 \mathrm{mM}$ and a $k_{\text {cat }}$ value of $0.96 \mathrm{~s}^{-1}$. Meanwhile, the optimum $\mathrm{pH}$ of E.C.-ALDC was 6.5, and the activity of E.C.-ALDC was activated by $\mathrm{Mn}^{2+}, \mathrm{Ba}^{2+}, \mathrm{Mg}^{2+}, \mathrm{Zn}^{2+}$ and $\mathrm{Ca}^{2+}$, while $\mathrm{Cu}^{2+}$ and $\mathrm{Fe}^{2+}$ significantly inhibited ALDC activity. More importantly, we solved and reported the first crystal structure of E.c.-ALDC at $2.4 \AA$ resolution. The active centre consists of a zinc ion coordinated by highly conserved histidines (199, 201 and 212) and glutamates (70 and 259). However, the conserved Arg150 in E.C.-ALDC orients away from the zinc ion in the active centre of the molecule, losing contact with the zinc ion. Molecular docking of the two enantiomers of $\alpha$-acetolactate, $(R)$ acetolactate and (S)-acetolactate allows us to further investigate the interaction networks of E.C.-ALDC with the unique conformation of Arg150. In the models, no direct contacts are observed between Arg150 and the substrates, which is unlikely to maintain the stabilization function of Arg150 in the catalytic reaction. The structure of E.C.-ALDC provides valuable information about its function, allowing a deeper understanding of the catalytic mechanism of ALDCs.

\section{Introduction}

In microbes, acetoin (3-hydroxy-2-butanone, AC) is the inbetween product of the 2,3-butanediol (BD) fermentation pathway. With the growing demand in recent years, the production method of AC has been widely addressed. At present, commercially available AC is mainly chemically synthesized from fossil materials, such as 2,3-butanediol (2,3$\mathrm{BD})$, butanone and diacetyl. However, due to environmental problems in chemical processes, large-scale production of AC is still limited. Based on microbial fermentation and enzymatic catalysis, biotechnological production provides an alternative environmentally friendly method, but the final product is usually a mixture. The mixture contains by-products, such as 2,3-BD, acetic acid and lactic acid, resulting in the difficult separation of AC in microbial fermentation. Usually, the

\footnotetext{
${ }^{a}$ School of Life Science and Biotechnology, Dalian University of Technology, Dalian, Liaoning, 116024, P. R. China. E-mail: fanglingji@dlut.edu.cn

${ }^{b}$ Marine Bioengineering Group, Dalian Institute of Chemical Physics, Chinese Academy of Sciences, Dalian, Liaoning, 116023, P. R. China. E-mail: xuesong@dicp.ac.cn ${ }^{c}$ School of Food and Environment Science and Engineering, Dalian University of Technology, Panjin, Liaoning, 12422, P. R. China

$\dagger$ These two authors contributing equally to the paper.
}

separation of high purity acetoin from the mixtures is very expensive.

To date, many bacterial strains, such as Bacillus subtilis, ${ }^{\mathbf{1}}$ Klebsiella pneumoniae ${ }^{2}$ Enterobacter aerogenes ${ }^{3}$ and Enterobacter cloacae, ${ }^{4}$ have been discovered and engineered to produce AC. Among these microorganisms, the most promising microorganism for biological production of AC is Enterobacter cloacae. Enterobacter cloacae subsp. dissolvens strain SDM grows rapidly in a simple medium and metabolizes the major lignocellulosederived sugars glucose and xylose into AC. ${ }^{4}$ However, AC exists in two stereoisomeric forms: $(R)$-AC and $(S)$-AC. The efficient use of AC, especially in the asymmetric synthesis of valuable chiral chemicals, requires optically active AC. However, owing to a lack of knowledge about stereoisomer formation mechanisms, the production of optically active AC using these strains has been difficult to achieve. Industrial chiral AC production by using these promising microorganisms has not been reported to date.

Three enzymes involved in AC biosynthesis from pyruvate through $\alpha$-acetolactate (AL) to AC include $\alpha$-acetolactate synthase (ALS, encoded by $b u d B), \alpha$-acetolactate decarboxylase (ALDC, encoded by budA), and acetoin reductases (ARs, also called DRs or BDHs, encoded by budC and $g d h$ ) (Scheme 1). ALDC (EC 4.1.1.5) catalyses $\alpha$-acetolactate to form AC and carbon dioxide via decarboxylation. ${ }^{5}$ In some cases, the 


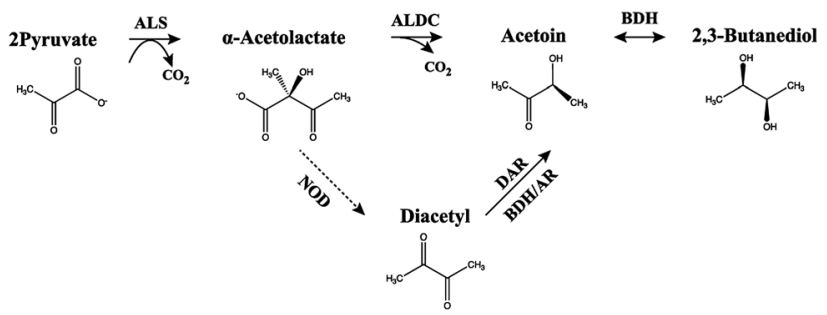

Scheme 1 Scheme of the acetolactate pathway starting with pyruvate. In the figure: ALS, $\alpha$-acetolactate synthase; ALDC, $\alpha$-acetolactate decarboxylase; $\mathrm{BDH}, 2,3$-butanediol dehydrogenase; $\mathrm{AR}$, acetoin reductase; DAR, diacetyl reductase and NOD, nonoxidize non-enzymatic oxidative decarboxylation.

decarboxylation of $\alpha$-acetolactate by ALDC is assumed to be a rate-limiting step in generating 2,3-BD. ${ }^{6,7}$ Immobilized ALDC has been applied in the traditional beer brewing process. ${ }^{8}$ During fermentation, ALDC significantly increases the production rate and decreases the adverse flavour caused by diacetyl. ${ }^{9}$ Apart from the catabolic degradation of $\alpha$-acetolactate to acetoin, ALDC also participates in the biosynthesis of branchedchain amino acids. ${ }^{\mathbf{1 0 - 1 2}}$

Up to now, the functionally characterized and structurally solved ALDCs are those from Bacillus subtilis (B.s.-ALDC), ${ }^{13}$ Bacillus brevis (B.b.-ALDC), ${ }^{\mathbf{1 4}}$ Brevibacillus brevis ${ }^{9}$ and Lactococcus lactis subsp. lactis. ${ }^{\mathbf{1 1}} \mathrm{X}$-ray crystal structures of ALDCs from Bacillus subtilis ${ }^{\mathbf{1 3}}$ and Bacillus brevis ${ }^{\mathbf{1 5}}$ show that both of the ALDCs feature a two-domain $\alpha / \beta$ tertiary structure, in which the $\mathrm{N}$-terminal domain is composed of a seven-stranded mixed $\beta$ sheet. In the asymmetric unit cell, the dimer assembly is composed of the seven-stranded mixed $\beta$-sheet, which extends into the equivalent $\beta$-sheet of the two-fold symmetry-related molecule, forming a fourteen-stranded $\beta$-sheet. The structures of a series of designed transition state analogues in complex with B.b.-ALDC suggest a catalytic mechanism. ALDC catalyses not only the decarboxylation of the favoured $(S)$-AL but also the isomerization via carboxyl migration and subsequent decarboxylation of the less-favoured $(R)$-substrate..$^{15}$ In addition, two groups ${ }^{16}$ simulated the catalytic processes using the hybrid quantum mechanical/molecular mechanical (QM/MM) calculations. Even though the amino sequence identities between B.b.-ALDC and B.s.-ALDC are very low (only 30\%), the active centre is well-conserved. By docking the two enantiomers of $\mathrm{AL}$ into the crystal structure of B.s.-ALDC, Ji et al. simulated the binding interaction of the substrates and investigated the substrate preference. A more stable binding of $(S)$-AL versus $(R)$ $\mathrm{AL}$ is revealed by the lower binding free energy. ${ }^{13}$

Due to less structural knowledge of ALDCs, the determination of the structural characteristics of the E.c.-ALDC is essential to obtain new insights into its catalytic reaction. We thus cloned, expressed and characterized the ALDC from Enterobacter cloacae. Here, we report the crystal structure of E.c.ALDC at a resolution of $2.4 \AA$, which provides an original view of the structural features of E.c.-ALDC and investigate the structure-function relationship of E.c.-ALDC. In the active site, the conserved His199, His201 and His212 residues preserve the overall structure of the ALDC, except Arg150, which orients away from the active site, forming hydrogen bonds with residues at the C-terminus. Additionally, Arg150 was proven to maintain the same conformation and showed no contacts with the substrates though molecular docking complexes of E.c.-ALDC with $(S)$-AL and $(R)$-AL. The fully characterized activities and structure of the enzyme provide insights into ALDCs for the bioproduction of acetoin.

\section{Results and discussion}

\section{Enzymatic and kinetic properties of E.c.-ALDC}

The activity of ALDC was previously studied using the VogesProskauer (VP) assay. ${ }^{29}$ However, it was challenging to obtain reproducible results using this assay since the substrate and the colour regent are not stable during the reaction. To avoid this problem, we carried out an enzymatic assay on B.s.-ALDC using CD as our research technique. ${ }^{13}$ The CD spectra of $(S)$-AL and $(R)$-AC are reported ${ }^{30-32}$ and are sufficiently different to allow for the monitoring of the enzyme activity assay. At the beginning, no CD signal was observed due to the racemic substrate, whereas the spectra for $(R)$-AL and $(R)$-AC appeared in addition to the enzyme. The signal for $(R)$-AL was observed because the enzymatic turnover of $(S)$-AL produces an excess of $(R)$-AL (Fig. 1A). Two wavelengths at $278 \mathrm{~nm}$ and $315 \mathrm{~nm}$ were identified to monitor the assay reaction. As presented in Marlow's work, at $278 \mathrm{~nm}$, the formation of $(R)$-AC can be monitored. At this point, the CD signal for $(S)$-AL crosses the axis and has no signal. Though the slope is negative because of the negative peak, but it is proportional to the formation rate of $(R)$-AC. At

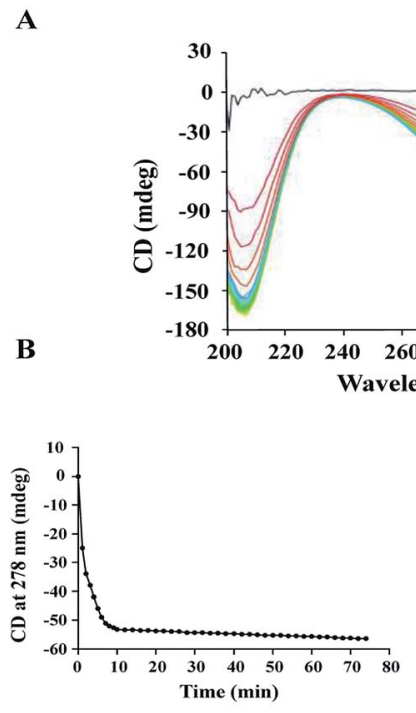

Fig. 1 Enzymatic properties of E.C.-ALDC measured by circular dichroism (CD). (A) E.C. -ALDC catalysed ( \pm )-AL monitored by continuous wavelength scan CD from $190 \mathrm{~nm}$ to $340 \mathrm{~nm}$. The entire spectra are shown starting from $( \pm)-\mathrm{AL}$ (blue) to $(R)-\mathrm{AC}$ (purple). Different colour represents different time points. (B) AC signal at 278 (left) and $315 \mathrm{~nm}$ (right). At $278 \mathrm{~nm}$, there is no signal for ( $S$ )-AL, and the changed $C D$ signal is due to the formation of $(R)-A C$. At $315 \mathrm{~nm}$, there is no signal for $(R)-A C$, and the changed $C D$ signal is due to the disappearance of (S)-AL. 
$315 \mathrm{~nm}$ the CD signal for $(R)$-AC is zero and only the tail of a positive $(S)$-AL peak is observed. This is the disappearance of a positive peak so the rate observed reflects the loss of $(S)$-AL over time. At $278 \mathrm{~nm}$, the formation of $(R)$-AL can be observed initially at a faster rate, followed by a slower rate (Fig. 1B, left). At $315 \mathrm{~nm}$, the $( \pm)$-AL signal initially started at zero, and the rapid formation of a negative peak formed due to an excess of $(R)$-AL from the enzymatic turnover of $(S)$-AL. Subsequently, the peak begins to disappear at a slower rate as the enzyme turnover for the remaining $(R)$-AL slows (Fig. 1B, right). As the signal for $(R)$-AC was stronger than that for $( \pm)$-AL, the activity was calculated using the signals at $278 \mathrm{~nm}$, and the specific activity of E.c.-ALDC ( \pm -AL was $1024.37 \mathrm{U} \mathrm{mg}^{-1}$. The rate was converted from units of mdeg $\mathrm{s}^{-1}$ into units of $\mathrm{mM} \mathrm{s}^{-1}$ by using molar ellipticity and then into specific activity with units of $\mu \mathrm{mol}$ $\mathrm{mg}^{-1} \min ^{-1}$.

Kinetic studies were conducted on E.c.-ALDC over a concentration range of $( \pm)$-AL. Kinetic data were calculated based on curve fitting to the Lineweaver-Burk double reciprocal curve for three replicates. The calculated Michaelis-Menten constant $\left(K_{\mathrm{m}}\right)$ and turnover number $\left(k_{\text {cat }}\right)$ values of E.c.-ALDC were $12.19 \mathrm{mM}$ and $0.96 \mathrm{~s}^{-1}$, respectively. In our previous work studying B.s.-ALDC, the $K_{\mathrm{m}}$ and $k_{\text {cat }}$ values of B.s.-ALDC were $21 \mathrm{mM}$ and $2.21 \mathrm{~s}^{-1} \cdot{ }^{13}$ However, in the kinetic analysis of B.s.ALDC, Marlow and his coworker ${ }^{15}$ simultaneously measured B.s.-ALDC kinetic parameters by the NADH method with $( \pm)$-AL and the CD method with $(S)$-AL, yielding $K_{\mathrm{m}}=0.25 \pm 0.08 \mathrm{mM}$ and $k_{\text {cat }}=5.99 \pm 0.95 \mathrm{~s}^{-1}$ for the former and $K_{\mathrm{m}}=4.31 \pm$ $1.14 \mathrm{mM}$ and $k_{\text {cat }}=29.59 \pm 2.96 \mathrm{~s}^{-1}$ for the latter. ALDC activity has been detected only among certain bacterial species, such as Bacillus sp. and Lactobacillus sp., ${ }^{33}$ and the ALDCs from different bacteria displayed different physical and chemical properties. In the thermophilic cell-free cascade enzymatic reaction for acetoin synthesis from pyruvate, Jia et al. used creatine and $\alpha$-naphthol with the $V_{\max }$ and $K_{\mathrm{m}}$ values for pyruvate by B.s.-ALDC were $2.34 \pm 0.03 \mu \mathrm{mol} \mathrm{min}{ }^{-1} \mathrm{mg}^{-1}$ and 60.51 $\pm 2.67 \mathrm{mM}$, respectively, under the optimum conditions. ${ }^{34}$

\section{Optimization of the decarboxylation conditions}

To test the impact of pH changing the enzyme activity of E.c.ALDC, the activity assays were carried out with $\mathrm{pH}$ levels ranging from 4.5 to 8.5 , and the results are presented in Fig. 2A. The highest activity of E.c.-ALDC enzyme was observed at $\mathrm{pH} 6.5$, and the activity was $963 \pm 28 \mathrm{U} \mathrm{mg}^{-1}$. At $\mathrm{pH}$ values ranging from 4.5 to 6.0 , the activity of E.c.-ALDC dropped dramatically. As indicated by the results, E.c.-ALDC activity is more effectively inhibited at an acidic $\mathrm{pH}$ than at an alkaline $\mathrm{pH}$. These results suggest that the secondary interaction (ionic and polar interactions, hydrogen bonding) within the enzyme was stable, resulting in improved ALDC tolerance to alkaline conditions. ${ }^{35}$ The ALDC from Bacillus subtilis exhibited the maximum activity at $\mathrm{pH} 5.0 .^{13}$ The ALDC from Lactobacillus casei DSM 2547 showed a relatively high activity at $\mathrm{pH} 4.5$ to $5.0 .^{36}$ The ALDC from Lactococcus lactis had an optimum $\mathrm{pH}$ of $\mathrm{pH} 6.0 .{ }^{37}$ Moreover, the electrostatic surface properties of E.c.-ALDC were investigated (Fig. 2B) with an environmental $\mathrm{pH}$ of 7.0. The
A

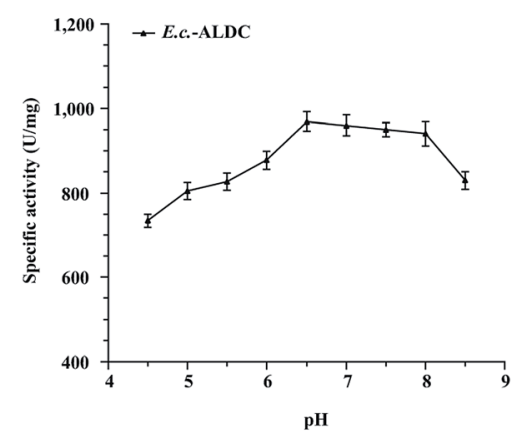

B
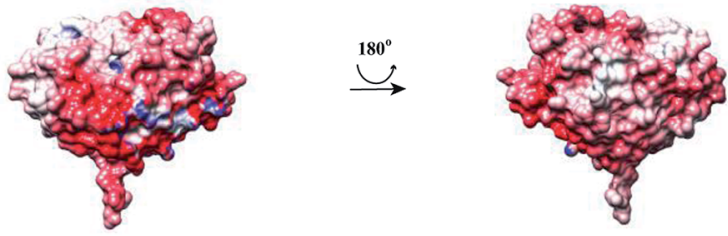

C

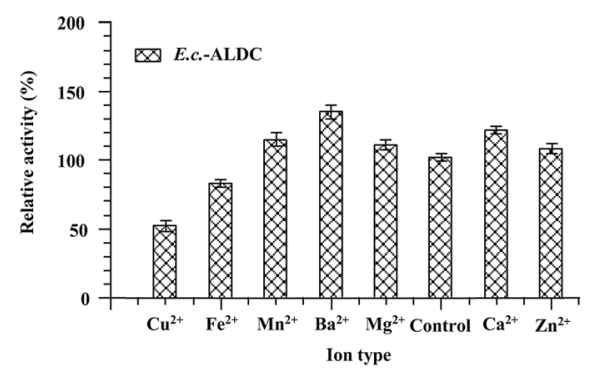

Fig. 2 Optimization of the catalytic conditions of E.C.-ALDC. (A) Effects of $\mathrm{pH}$ on the enzyme activity of E.c.-ALDC. Error bars indicate standard deviations of three independent experiments. (B) Representation of the electrostatic potential at the surface of E.C.-ALDC in two orientations. The protein is shown as solvent-accessible surface coloured by electrostatic potential at $\pm 5 \mathrm{kT}^{-1}$. Color-codes depend on the electrostatic potential (red: negative charge; blue, positive charge; and white: neutral charge). (C) Effects of different metal ions on the enzyme activity of E.c.-ALDC. Error bars indicate standard deviations from three independent experiments.

calculated theoretical isoelectric point (pI) of E.c.-ALDC was 5.4. The E.c.-ALDC revealed two differently charged faces. One face was mostly neutral with two negatively charged areas, while the second face was highly negatively charged.

As is reported in the crystal structures of ALDCs, $\mathrm{Zn}^{2+}$ plays a very important role in stabilizing the substrates and is involved in electron transfer. The effects of various metal ions on E.c.-ALDC were investigated. EDTA was used to chelate the metal ions of E.c.-ALDC, which were then measured by an inductively coupled plasma (ICP) technique. As indicated in Fig. 2C, $\mathrm{Mn}^{2+}, \mathrm{Ba}^{2+}, \mathrm{Mg}^{2+}, \mathrm{Zn}^{2+}$ and $\mathrm{Ca}^{2+}$ greatly improved the activity of E.c.-ALDC, while $\mathrm{Fe}^{2+}$ inhibited the activity of E.c.ALDC. $\mathrm{Cu}^{2+}$ significantly decreased the activity of E.c.-ALDC. In fact, the function of many decarboxylases requires a divalent ion as a cofactor. ALDC from Lactococcus lactis DX was activated by $\mathrm{Fe}^{2+}, \mathrm{Zn}^{2+}, \mathrm{Mg}^{2+}, \mathrm{Ba}^{2+}$ and $\mathrm{Ca}^{2+}$ and was significantly inhibited by $\mathrm{Cu}^{2+} \cdot{ }^{38}$ Some of the metal ions do not function as catalytic centres. For example, the $\mathrm{Zn}^{2+}$ ion in the biotin- 
dependent decarboxylases helped to properly orient the substrate, while the $\mathrm{Mg}^{2+}$ ion in the ThDP-dependent decarboxylases assisted in binding the thiamine cofactor. ${ }^{39,40}$ For $\mathrm{NAD}(\mathrm{P})+-$-dependent decarboxylases, such as malic enzymes, a divalent metal ion $\left(\mathrm{Mn}^{2+}\right.$ or $\left.\mathrm{Mg}^{2+}\right)$ presented significant influences on both catalysis and structural stability. ${ }^{41}$

\section{Overall structure of E.c.-ALDC}

The crystal structure of E.c.-ALDC comprises two domains of $\alpha /$ $\beta$ tertiary structure, in which each domain contains a sevenstranded mixed $\beta$-sheet. This is essentially the same as the already described crystal structures of B.s.-ALDC ${ }^{13}$ and B.b.ALDC. $^{42}$ These two $\beta$-sheets form a nearly parallel intramolecular surface (back-to-back) (Fig. 3A). In-between this surface, the shortest distance of all $\mathrm{C}_{\alpha}$ atoms of the backbone chain is $6.2 \AA$ between Ile72 and His201. The N-terminal domain consists of a seven-stranded mixed $\beta$-sheet, whereas the C-terminal domain consists of a five-stranded $\beta$-sheet, two of which elongate with a 180 degree turn generating two $\beta$-sheet strands. Two ALDC molecules are present in the asymmetric unit, and they exhibit an average pairwise backbone atom root mean square deviation (RMSD) value of $0.26 \AA$, exhibiting highly structural consistency. The seven-stranded mixed $\beta$-sheet on the $\mathrm{N}$-terminal domain of E.c.-ALDC extends into the equivalent $\beta$-sheet of the two-fold symmetry-related molecule, resulting in a fourteen-stranded $\beta$-sheet that spans the physiologically relevant dimeric assembly, with the same structures as B.b.-ALDC ${ }^{42}$ and B.s.-ALDC, ${ }^{15}$ which might shed light on the dimer formation (Fig. 3A).

In the structure of E.c.-ALDC, the electron density for the active centre residues is well resolved (Fig. 3B). $\mathrm{A} \mathrm{Zn}^{2+}$ ion coordinated by His199, His201 and His212 together with Glu259 from the C-terminal tail, and these residues are highly conserved in ALDCs (Fig. 2B). In addition, the other two highly conserved Glu70 and Arg150 are also presented in this structure. In the catalytic domain of B.s.-ALDC, molecular docking results exhibit a similar conservative structure, a $\mathrm{Zn}^{2+}$ ion coordinated by His191, His193 and His201. Glu251, Glu62 and Arg142 interact with the substrate through hydrogen bonds. Three histidine-coordination of a metal ion is widely found in the structures of enzymes, for instance, manganese in oxalate
A

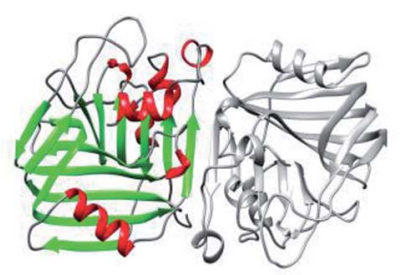

B

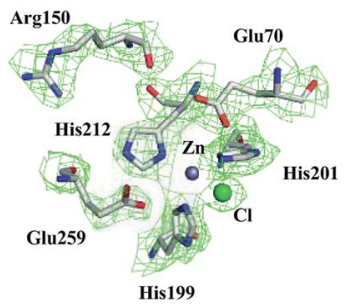

Fig. 3 3D structure of E.C.-ALDC. (A) The overall fold of E.C.-ALDC is shown as a ribbon diagram. (B) The electron densities of His199, His201 and His212; Glu70 and Glu255; and Arg150, which are in contact with a zinc ion within a $5 \AA$ distance. decarboxylase, ${ }^{5}$ copper in quercetin 2,3-dioxygenase ${ }^{43}$ and zinc in carbonic anhydrase. ${ }^{44}$ In the transition state, analogue structures of B.b.-ALDC with (2S,3S)-2,3-dihydroxy-2methylbutanoic acids (PDB ID: 4BT4) and (2S,3R)-2,3dihydroxy-2-methylbutanoic acids (PDB ID: 4BT5), the Glu65, Arg145, and Glu253 residues contact the analogues through hydrogen bonds, and the inhibitors adopt essentially identical conformations. Meanwhile, Glu65 and Arg145 also form stabilizing hydrogen bonds with the analogues. ${ }^{15}$

\section{Comparisons of ALDC structures}

We compared the structures of E.c.-ALDC, B.b.-ALDC (PDB ID: 4BT2) and B.s.-ALDC (PDB ID: 5NXE). Despite the low sequence identities of E.c.-ALDC with B.s.-ALDC and B.b.-ALDC, the superimposition of the overall structure of E.c.-ALDC onto that of B.b.-ALDC (Fig. 4B, upper left) and B.s.-ALDC (Fig. 4B, upper right) yields an average pairwise RMSD value of $0.712 \AA$ and $0.752 \AA$, respectively, for the backbone atoms. The evolutionary tree of these three ALDCs is shown in Fig. 4A. The low RMSD
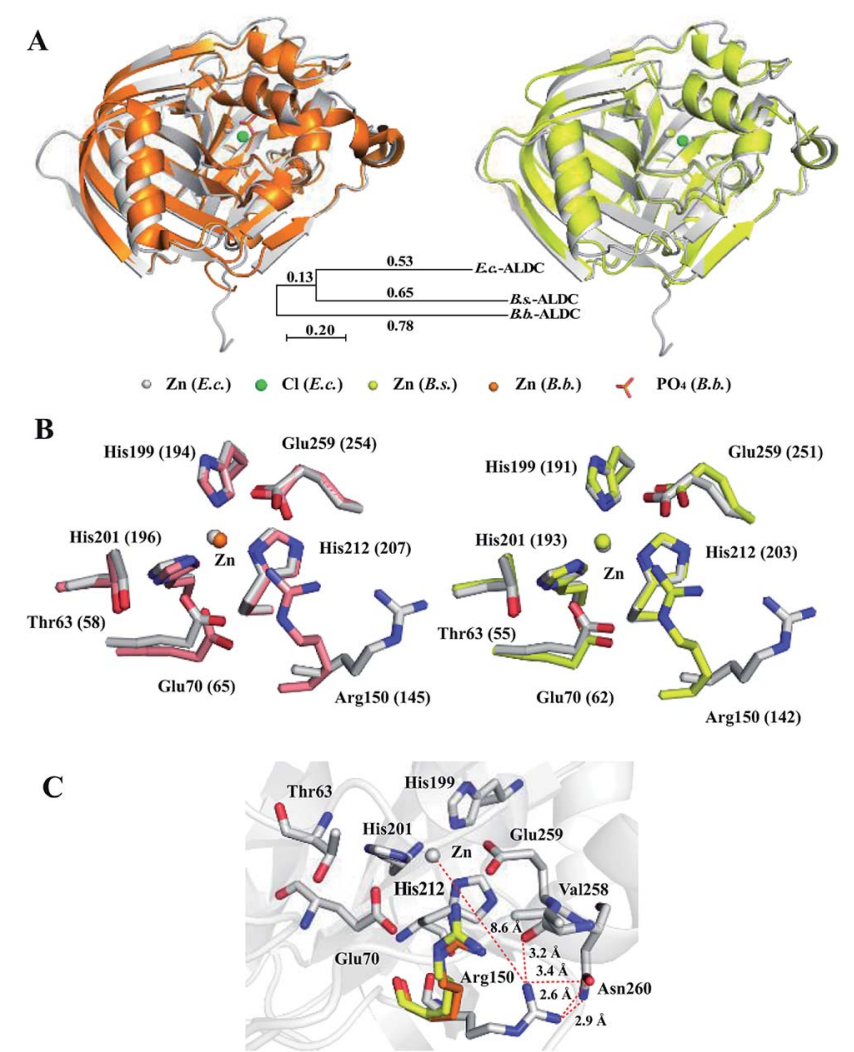

Fig. 4 Crystal structure comparisons within E.C.-ALDC, B.s.-ALDC and B.b.-ALDC. (A) The crystal structure of E.C.-ALDC (grey) is superimposed onto B.b.-ALDC (pink, PDB ID: 4BT7) and B.s.-ALDC (yellow, PDB ID: 5XNE). (B) Active site superimposed between E.c.-ALDC and B.b.-ALDC (pink, PDB ID: 4BT7) and between E.c.-ALDC and B.s.ALDC (yellow, PDB ID: 5XNE), including Thr63, His199, His201, His212, Glu70, Glu255, and Arg150. (B.b.-ALDC: Thr58, His194, His196, His207, Glu65, Glu254, and Arg145), (B.s.-ALDC: Thr55, His191, His193, His204, Glu62, Glu251, and Arg142). (C) Intramolecular interactions of Arg150 (grey, E.C.-ALDC; Arg145, pink, B.b.-ALDC; Arg142, yellow, B.s.-ALDC) with Val258, Glu259 and Asn260. 
value indicates that the overall structure of the E.c.-ALDC is similar to the structures of B.b.-ALDC and B.s.-ALDC. We also superimposed the highly conserved amino acid residues in the active site (His199, His201 His212, Glu70, Glu259, Arg150 and Thr63) onto those of B.b.-ALDC (Fig. 4B, lower left) and B.s.ALDC (Fig. 4B, lower right). Apart from Arg150, the coordinates of the side chains of the remaining residues overlay well.

The orientation of the side chain of Arg150 in the structure of E.c.-ALDC differs from the arginine in B.s.-ALDC and in B.b.ALDC. In E.c.-ALDC, the side chain of Arg150 orients away from the active site centre with an angle of 50 degrees. In addition to the highly conserved Thr58 and Glu65 in B.b.-ALDC, arginine at the corresponding site is also highly conserved in the vicinity of the metal. Likewise, in E.c.-ALDC, the distance between the zinc ion and the $\mathrm{NH} 2$ group of the side chain of Arg150 is $8.6 \AA$; however, in B.s.-ALDC and B.b.-ALDC, this distance is extremely smaller, $4.0 \AA$ and $3.9 \AA$, respectively. In fact, both of the $\mathrm{NH}_{2}$ groups in the side chain of the arginine residue (Arg142 in B.s.ALDC and Arg145 in B.b.-ALDC) forms hydrogen bonds with the oxygen atom of the glutamate residue (Glu250 in B.s.-ALDC and Glu253 in B.b.-ALDC). In E.c.-ALDC, the $\mathrm{NH}_{2}$ group of Arg150 forms a hydrogen bond with the oxygen atom of the backbone of Val258 (3.2 ̊). The $\mathrm{NH}_{2}$ group of Arg150 is in close proximity to the ND2 group of Asn260 with a distance of $2.9 \AA$ (Fig. 4C). It is possible that the unique conformation of Arg150 causes a loss of function in stabilizing the substrate.

The glutamate at the active centre plays a significant role during the decarboxylation reaction. The highly conserved Glu259 in E.c.-ALDC conducted a similar structure conformation as the corresponding glutamate structure in B.s.-ALDC and $B$. $b$.-ALDC. The distance between the carbonyl group of the side chain and the zinc ion of Glu259 is $3.6 \AA$, while in the structures of B.s.-ALDC and B.b.-ALDC, the distances are $3.5 \AA$ and $4.0 \AA$, respectively. In the crystal structure of B.b.-ALDC in complex with transient intermediate analogues, glutamate forms a hydrogen bond with the analogues. ${ }^{15}$ Meanwhile, in the docking models of B.b.-ALDC with $(S)$-AL, the carboxyl group of Glu253 forms a hydrogen bond with Arg145 in the active site. ${ }^{9}$ In the rearrangement process, the hydrogen bond between Glu253 and the carboxyl of $(R)$-AL is critical when the carboxyl rearranges to the adjacent carbonyl carbon to form $(S)$-AL. ${ }^{16}$

\section{Molecular docking with substrate}

The complex structures of E.c.-ALDC with $(S)$-AL and $(R)$-AL were prepared on the basis of crystal structures of B.b.-ALDC in complex with two transition state analogues. The energy optimized structures are displayed in Fig. 5. In the complex structure of $(S)$-AL and E.c.-ALDC, the $\mathrm{Zn}^{2+}$ ion forms a pentacoordinated structure with the nearby His199, His201 and His212 at distances of 1.9, 2.0 and $2.2 \AA$, respectively, and with the oxygen atoms on the carboxyl and hydroxyl groups of $(S)$-AL at distances of 1.9 and $2.7 \AA$, respectively; this indicates a strong coordination interaction of the $\mathrm{Zn}^{2+}$ ion with the three histidine residues and the substrate (Fig. 5A). These interaction networks are also presented in the models of B.s.-ALDC with $(S)$ $\mathrm{AL}^{13}$ and B.b.-ALDC with $(S)-\mathrm{AL}^{9,16}$ via the molecular docking
A
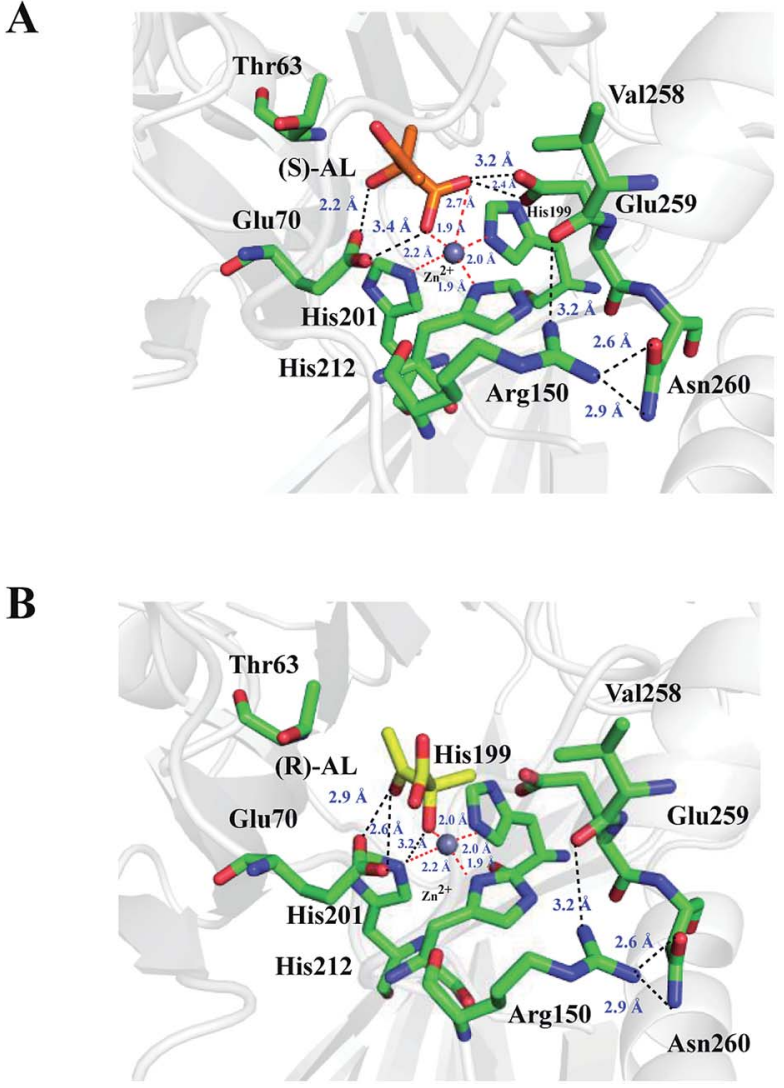

Fig. 5 Energy optimized active site structures of E.C.-ALDC in complex with the $(S)-A L$ (A, orange) and $(R)-A L(B$, yellow). Selected key distances are shown in angstroms. Hydrogen bonds are shown in black dashed lines, and coordination bonds are shown in red dashed lines.

method. The role of the $\mathrm{Zn}$ (II), as a Lewis acid, is best understood in terms of the reverse reaction of acetoin carboxylation. The mode of binding for the $(R, R)$-dihydroxy-2-methylbutanoic acids (PDB ID: 4BT5) isomer supports the proposed decarboxylation mechanism for the natural $(S)$-enantiomer, also involving transient binding of the departing $\mathrm{CO}_{2}$ to the zinc ion. In addition, the side chain of Glu70 (Glu65 in B.s.-ALDC and Glu62 in B.b.-ALDC) has polar interactions with $(S)$-AL. Likewise, Arg150 maintains the interactions with Val258, Glu259 and Asn260 as in the apo structure of E.c.-ALDC and did not change its unique conformation in the presence of $(S)$-AL. Thus, Arg150 is not able to preserve the critical role in stabilizing the $\mathrm{Zn}^{2+}$ ion, which differs from the corresponding arginines in B.b.-ALDC and B.s.-ALDC. In the transition state, analogue structures of B.b.-ALDC in complex with $(2 S, 2 S)$-2,3-dihydroxy-2methylbutanoic acid (PDB ID: 4BT4) and (2S,2R)-2,3-dihydroxy2-methylbutanoic acid, Arg145 contacts the analogues through hydrogen bonds, and the inhibitors adopt essentially identical conformations. Arg145 is well positioned to potentially assist delivery of the proton, which creates a new chiral centre in B.b.ALDC. ${ }^{15}$ In the docking model of B.s.-ALDC with $(S) /(R)$-AL, a hydrogen bond between the $\mathrm{OH}$ of $(R)$-AL and the side chain of Arg142 is observed, while no hydrogen bond formed in B.s.ALDC with $(S)$-AL.13 In the transition state of analogue 
structures of B.b.-ALDC in complex with $(2 S, 2 S)$-dihydroxy-2methylbutanoic acid (PDB ID: 4BT4) and (2S,2R)-dihydroxy-2methylbutanoic acid, Arg145 contacts the analogues through hydrogen bonds, and the inhibitors adopt essentially identical conformations.

The binding mode of E.c.-ALDC with (R)-AL slightly differs from the binding mode of $(S)$-AL. As shown in Fig. 5B, $(R)-\mathrm{AL}$ adopts a perpendicular conformation to that of $(S)$-AL in E.c.ALDC with its polar groups facing Glu259. The hydroxyl and carboxyl groups of $(R)$-AL are further from Glu259, forming hydrogen bonds with the OE1 and OE2 groups of Glu259 at a distance of 2.7 and $4.2 \AA$, respectively. Thus, the $\mathrm{Zn}^{2+}$ ion only forms a tetracoordinated structure with His199, His201, His212 and the substrate, with binding distances of 1.9, 2.0, 2.2 and 2.0 $\AA$, respectively. These hydrogen bonds stabilize the substrate in the active site of the enzyme. Arg150 in this model exhibits no conformational changes without interacting with $(R)$-AL. Again, Arg150 is unlikely to stabilize the substrate or deliver the proton in the catalytic function of E.c.-ALDC. To our knowledge, $(R)$-AL is not the natural substrate of ALDCs, while $(S)$-AL is. Correspondingly, in the cocrystallized complexes of B.b.-ALDC with the substrate analogues, the promoting carboxylate rearrangement mechanism of $(R)$-AL into $(S)$-AL was observed and proposed. ${ }^{42}$

\section{Experimental}

\section{Materials and methods}

Expression and purification of E.c.-ALDC. Protein was extracted essentially based on procedures previously reported for ALDCs. ${ }^{13}$ The genome of Enterobacter cloacae CICC 10011 was used as a template to clone the gene of E.c.-ALDC (bud A) and inserted into the expression vector pET-21b. E. coli BL21 (DE3) harbouring pET-21b with the E.c.-ALDC gene were grown at $37{ }^{\circ} \mathrm{C}$ and induced for $5 \mathrm{~h}$. E. coli cells were then harvested, and the pellet was resuspended in $20 \mathrm{mM}$ tris(hydroxymethyl) aminomethane (Tris) buffer ( $\mathrm{pH} 8.0$ ), containing $1 \mathrm{mM}$ dithiothreitol (DTT), $1 \mathrm{mM}$ ethylenediaminetetraacetic acid (EDTA) and $20 \%$ glycerol, followed by lysis by sonication on ice at the power of $400 \mathrm{~W}$ for $10 \mathrm{~min}$. The supernatant was loaded onto a pre-equilibrated HiTrap DEAE FF anion exchange column (GE Healthcare) in $20 \mathrm{mM}$ Tris buffer ( $\mathrm{pH}$ 8.0) containing $1 \mathrm{mM}$ DTT. The column was then eluted using a linear $\mathrm{NaCl}$ gradient from 0 to $500 \mathrm{mM}$ over 20 column volumes. Fractions containing the protein were collected, concentrated and subjected to gel filtration on a Superdex 200 16/60 column (GE Healthcare) in $20 \mathrm{mM}$ Tris buffer ( $\mathrm{pH} 8.0$ ) containing $1 \mathrm{mM}$ DTT. The purified protein was confirmed by SDS-PAGE, and the protein concentration was determined using the Bradford protein assay method.

Enzyme activity assay. For the activity assay of E.c.-ALDC, the measurements were based on procedures previously reported for B.s.-ALDCs, ${ }^{13}$ using circular dichroism (CD, JASCO J-810) to study the activity and kinetics of E.c.-ALDC. ${ }^{15}$ Freshly prepared substrate $( \pm)$-AL was obtained by diluting ( \pm )-ethyl 2-acetoxy-2methylacetoacetate (Aldrich) with $0.5 \mathrm{M} \mathrm{NaOH}$ and stirring for $20 \mathrm{~min}$ at room temperature. The mixture was adjusted to $\mathrm{pH}$
6.0 by the addition of $\mathrm{HCl}$ and then diluted with $10 \mathrm{mM}$ Tris$\mathrm{HCl}$ buffer ( $\mathrm{pH}$ 6.0) to the desired concentration. To generate the standard curve of $(R)-\mathrm{AC}$, with the complete reaction of $( \pm)$-AL with E.c.-ALDC, the produced stock solution was diluted to $0.5,1.0,5.0,10.0,15.0$ and $0.5 \mathrm{mM}$. The standard curve of $(R)$ AC was measured by CD using the following parameters: the scanning range 200-340 nm, data pitch $1 \mathrm{~nm}$, response $0.5 \mathrm{~s}$, bandwidth $2 \mathrm{~nm}$ and scanning speed $200 \mathrm{~nm} \min ^{-1}$. Using a cuvette with an optical path length of $1 \mathrm{~mm}$, an equal volume of substrate and E.c.-ALDC were mixed to start the reaction. The subsequent decarboxylation of $( \pm)$-AL into $(R)$-AC was monitored, and the CD signal at $278 \mathrm{~nm}$ was recorded. The amount of AC produced by ALDC was measured against the $(R)$-AC standard curve, resulting in the activity of E.c.-ALDC. One unit of E.c.-ALDC activity corresponds to the amount of E.c.-ALDC required for the formation of $1 \mu \mathrm{mol} \mathrm{AC}$ per minute at $30{ }^{\circ} \mathrm{C}$.

The kinetic parameters ( $K_{\mathrm{m}}$ and $k_{\text {cat }}$ values) of E.c.-ALDC were calculated by measuring enzyme activity using various initial concentrations $(53.4,26.7,17.8,13.3,10.7,8.9,7.6,6.7,5.9$ and $5.3 \mathrm{mM})$ of $( \pm)$-AL at $30{ }^{\circ} \mathrm{C}$ and $\mathrm{pH} 6$ and fitting to a double reciprocal curve by a Lineweaver-Burk plot. The process was monitored by CD as described above.

Optimum $\mathrm{pH}$ and ion determination. The optimal $\mathrm{pH}$ of E.c.ALDC was determined in buffers with different $\mathrm{pH}$ values (citrate phosphate 4.5-6.5, Tris 6.0-8.5) at $30^{\circ}$ C. E.c.-ALDC was preincubated with these buffers at $4{ }^{\circ} \mathrm{C}$ for an hour, and then the activity of E.c.-ALDC was determined using the CD abovementioned method at a temperature of $30{ }^{\circ} \mathrm{C}$. Moreover, the electrostatic surface potentials of E.c.-ALDC were calculated by $\mathrm{APBS}^{17}$ using an ionic strength of $0.15 \mathrm{M}$. The PDB2PQR server ${ }^{18}$ was used to prepare the structure for electrostatic calculations. Figures were generated using PYMOL 1.5.0.3. ${ }^{19}$ In addition, the effect of different metal ions on the activity of E.c.-ALDC was analysed with $1 \mathrm{mM} \mathrm{Cu}^{2+}, \mathrm{Fe}^{2+}, \mathrm{Mn}^{2+}, \mathrm{Ba}^{2+}, \mathrm{Mg}^{2+}, \mathrm{Zn}^{2+}$ and $\mathrm{Ca}^{2+}$ by the $\mathrm{CD}$ method at $30{ }^{\circ} \mathrm{C}$. In advance, E.c.-ALDC was treated with $0.5 \mathrm{mg} \mathrm{mL}{ }^{-1}$ EDTA, and the residual metal ions were measured by inductively coupled plasma emission spectrometry (ICP-ES).

Crystallization. Commercially available crystallization reagents, such as Index (Hampton Research) and Wizard (Agilent), were used to set up crystal screening. The initial crystallization trials of E.c.-ALDC were performed using a Mosquito crystallization robot (TTP LabTech) to set up sitting drops. The drops were composed of $0.1 \mu \mathrm{L}$ protein solution mixed with an equal volume of reservoir solution equilibrated against $40 \mu \mathrm{L}$ of the reservoir solution. Small crystals were observed after 2 weeks in the plates at $4{ }^{\circ} \mathrm{C}$. The final crystal for the diffraction collection is from a crystal grown in $0.1 \mathrm{M}$ sodium acetate trihydrate, $\mathrm{pH} 4.5,3.0 \mathrm{M}$ sodium chloride at a protein concentration of $30 \mathrm{mg} \mathrm{mL}^{-1}$ at $4{ }^{\circ} \mathrm{C}$.

$\mathrm{X}$-ray data collection and processing. Crystals of E.c.-ALDC grown in the above conditions were cryoprotected by incubation for $30 \mathrm{~s}$ in a solution containing glycerol (15\%) in $0.1 \mathrm{M}$ sodium acetate trihydrate, $\mathrm{pH}$ 4.5, 3.0 $\mathrm{M}$ sodium chloride prior to flashcooling in liquid nitrogen. Datasets were collected at the Shanghai Synchrotron Radiation Facility (SSRF) on beamline BL18U1. After the optimization of the procedures, 360 
diffraction images were collected using an MX225 charged coupled device (CCD) detector at $100 \mathrm{~K}$ with an oscillation range of $1.0^{\circ}$ and an exposure time of $1 \mathrm{~s}$ per frame, with a nonattenuated beam of $0.98 \AA \mathrm{X}$-ray wavelength. All diffraction data were processed, integrated, and scaled using the HKL-3000 software package. ${ }^{20}$ The structure was solved by molecular replacement (MR) with Phaser $^{21}$ using the coordinates of the catalytic domain of the same protein as an initial search model (Protein Data Bank (PDB) ID 5XNE). The model was improved by alternating cycles of manual model building and automated refinement using $\operatorname{Coot}^{22}$ and refined using REFMAC5 ${ }^{23}$ and PHENIX. $^{24}$ The stereochemical quality of the final model was assessed with MolProbity. ${ }^{25}$ In total, $96 \%$ and $4 \%$ of all residues are located in the favoured and allowed regions of the Ramachandran plot, respectively, and no residues were found in the disallowed region. The atomic coordinates and structure factor for E.c.-ALDC have been deposited in the Protein Data Bank (accession code 5YHO). All structure figures were prepared using PyMOL. ${ }^{26}$ A summary of the data-collection processing and structure-refinement statistics is given in Table 1.

Docking studies of $\boldsymbol{E} . \boldsymbol{c}$. -ALDC with $(\boldsymbol{S}) /(\boldsymbol{R})$-AL. Similar docking investigation of E.c.-ALDC with the substrates was conducted as the previous procedures in AutoDock 4.20 (http:// www.autodock.scripps.edu/). ${ }^{13}$ The empirical free energy function and the Lamarckian genetic algorithm were employed to identify the binding sites of $(S) /(R)$-AL with E.c.-ALDC. ${ }^{27,28}$ The computational models used in this work were constructed on

Table 1 Data collection and refinement statistics for E.C.-ALDC ${ }^{a}$

\section{Data collection}

Space group

$P 1211$

\section{Cell dimensions}

$a, b, c(\AA)$

$\alpha, \beta, \gamma\left({ }^{\circ}\right)$

Total reflections

Unique reflections

Resolution $(\AA)$

$R_{\text {merge }}$

$\langle I / \sigma I\rangle$

Completeness (\%)

$\langle$ Redundancy〉

$45.832,59.981,80.818$
$90.00,108.17,90.00$
$64798(6054)$
$16057(1551)$
$47.27-2.40$
0.075
23.47
97.69
4.0

Refinement

Resolution $(\AA)$

No. reflections

$R_{\text {work }} / R_{\text {free }}$

No. atoms

Protein

Water

3742

111

B-factors

Protein

Water

40.20

37.00

RMSD

Bond lengths $(\AA)$

Bond angles $\left({ }^{\circ}\right)$

0.004

0.97
${ }^{a}$ Values for the highest resolution bin are given in parentheses.
(1538)

$(0.223 / 0.251)$ the basis of the crystal structures of B.b.-ALDC in complex with the two transition state analogues (PDB ID: 4BT4 and 4BT5). Input structures of E.c.-ALDC and $(S) /(R)$-AL for docking simulation were stored in the PDBQT file format of AutoDock 4.20. The ligand input files were prepared according to the standard protocols in the AutoDock manual to prepare the protein target files. The default grid size was $60 \times 60 \times 60$ points, and the spacing was $0.375 \AA$, which corresponds to a cube with an edge length of $22.5 \AA$. The centre of the grid box was defined as the centre of the cocrystallized ligand. All the above procedures were performed using AutoDock Tools.

\section{Conclusions}

In this study, we report the enzymatic characterizations and original structure of ALDC from Enterobacter cloacae. Despite its high affinity for substrates at a $K_{\mathrm{m}}$ of $12.19 \mathrm{mM}$, the enzyme presented low catalytic efficiency. The structure of E.c.-ALDC preserved the typical conservative conformation of known ALDCs. Surprisingly, Arg150 exhibits a tilted orientation away from the active centre and loses its interaction with the zinc ion. Furthermore, by docking the substrates into the structure of E.c.-ALDC, no direct contacts are observed between Arg150 and the substrates. Thus, we believe that the unique conformation of Arg150 might explain the lower enzymatic activity of E.c.ALDC compared to that of the previously characterized ALDCs. However, further experimental cocrystallization studies are needed in the future to prove our hypothesis and in silico calculations. Our E.c.-ALDC structure provides very important clues to the catalytic mechanism of ALDCs.

\section{Conflicts of interest}

There are no conflicts of interest to declare.

\section{Acknowledgements}

Feida Long helped with the docking work. Additionally, we would like to thank the financial support from the National Science Foundation of China with grant number 21506025 and the Dalian University of Technology Science Foundation with grant number DUT8LK08.

\section{Notes and references}

1 B. Zhang, X. L. Li, J. Fu, N. Li, Z. Wang, Y. J. Tang and T. Chen, PLoS One, 2016, 11, e0159298.

2 J. W. Jang, H. M. Jung, D. G. Kim and M. K. Oh, Korean Chem. Eng. Res., 2017, 55, 237-241.

3 J. W. Jang, H. M. Jung, D. K. Im, M. Y. Jung and M. K. Oh, Enzyme Microb. Technol., 2017, 106, 114.

4 L. Zhang, Q. Liu, Y. Ge, L. Li, C. Gao, P. Xu and C. Ma, Green Chem., 2016, 18, 1560-1570.

5 R. Anand, P. C. Dorrestein, C. Kinsland, T. P. Begley and S. E. Ealick, Biochemistry, 2002, 41, 7659-7669.

6 S. Li, X. Gao, N. Xu, L. Liu and J. Chen, Microb. Cell Fact., 2014, 13, 55. 
7 M. H. Choi, S. J. Kim, J. W. Kim, H. J. Sim, Y. C. Park and J. H. Seo, Bioresour. Technol., 2016, 51, 170-176.

8 S. Qian, C. Wang, H. Wang, F. Yu, C. Zhang and H. Yu, Process Biochem., 2015, 50, 1388-1393.

9 C. Zhao, H. Su and Y. Liu, RSC Adv., 2016, 6, 80621-80629.

10 N. N. M. C. Renna, L. R. Winik and S. A. Zahler, J. Bacteriol., 1993, 175, 3863-3875.

11 N. Goupilfeuillerat, M. Cocaignbousquet, J. J. Godon, S. D. Ehrlich and P. Renault, J. Bacteriol., 1997, 179, 62856293.

12 C. Monnet, M. Nardi, P. Hols, M. Gulea, G. Corrieu and V. Monnet, Lett. Appl. Microbiol., 2010, 36, 399-405.

13 F. Ji, M. Li, Y. Feng, S. Wu, T. Wang, Z. Pu, J. Wang, Y. Yang, S. Xue and Y. Bao, Appl. Microbiol. Biotechnol., 2018, 102, 113.

14 S. Najmudin, J. T. Andersen, S. A. Patkar, T. V. Borchert, D. H. G. Crout and V. Fülöp, Acta Crystallogr., 2003, 59, 1073-1075.

15 V. A. Marlow, D. Rea, S. Najmudin, M. Wills and V. Fueloep, ACS Chem. Biol., 2013, 8, 2339-2344.

16 C.-B. Zhuang and Q.-C. Zheng, RSC Adv., 2016, 6, 9185291859.

17 N. A. Baker, D. Sept, S. Joseph, M. J. Holst and J. A. Mccammon, Proc. Natl. Acad. Sci. U. S. A., 2001, 98, 10037-10041.

18 T. J. Dolinsky, J. E. Nielsen, J. A. Mccammon and N. A. Baker, Nucleic Acids Res., 2004, 32, 665-667.

19 W. L. Delano, The PyMOL User's Manual, DeLano Scientific, San Carlos, CA, USA, 2002.

20 Z. Otwinowski and W. Minor, Macromolecular Crystallography, Pt A, 1997, vol. 276, pp. 307-326.

21 A. J. McCoy, R. W. Grosse-Kunstleve, P. D. Adams, M. D. Winn, L. C. Storoni and R. J. Read, J. Appl. Crystallogr., 2007, 40, 658-674.

22 P. Emsley, B. Lohkamp, W. G. Scott and K. Cowtan, Acta Crystallogr., 2010, 66, 486-501.

23 G. N. Murshudov, P. Skubák, A. A. Lebedev, N. S. Pannu, R. A. Steiner, R. A. Nicholls, M. D. Winn, F. Long and A. A. Vagin, Acta Crystallogr., 2011, 67, 355-367.

24 P. D. Adams, P. V. Afonine, G. Bunkóczi, V. B. Chen, I. W. Davis, N. Echols, J. J. Headd, L. W. Hung, G. J. Kapral and R. W. Grosse-Kunstleve, Acta Crystallogr., Sect. D: Biol. Crystallogr., 2010, 66, 213-221.
25 V. B. Chen, W. B. A. Iii, J. J. Headd, D. A. Keedy, R. M. Immormino, G. J. Kapral, L. W. Murray, J. S. Richardson and D. C. Richardson, Acta Crystallogr., 2010, 66, 12-21.

26 Schrodinger, LLC, The PyMOL Molecular Graphics System, Version 1.8., 2015.

27 M. F. Sanner, J. Mol. Graphics Modell., 1999, 17, 57-61.

28 G. M. Morris, D. S. Goodsell, R. S. Halliday, R. Huey, W. E. Hart, R. K. Belew and A. J. Olson, J. Comput. Chem., 2015, 19, 1639-1662.

29 B. Diderichsen, U. Wedsted, L. Hedegaard, B. R. Jensen and C. Sjøholm, J. Bacteriol., 1990, 172, 4315.

30 D. H. G. Crout and S. M. Morrey, J. Chem. Soc., Perkin Trans. 1, 1983, 2435-2440, DOI: 10.1039/P19830002435.

31 A. F. Drake, G. Siligardi, D. H. G. Crout and D. L. Rathbone, J. Chem. Soc., Chem. Commun., 1987, 24, 1834-1835.

32 M. Vinogradov, A. Kaplun, M. Vyazmensky, S. Engel, R. Golbik, K. Tittmann, K. Uhlemann, L. Meshalkina, Z. Barak and G. Hübner, Anal. Biochem., 2005, 342, 126-133. 33 S. E. Godtfredsen, H. Lorck and P. Sigsgaard, Carlsberg Res. Commun., 1983, 48, 239-247.

34 X. Jia, Y. Liu and Y. Han, Sci. Rep., 2017, 7, 4333.

35 S. L. Hosseinipour, M. S. Khiabani, H. Hamishehkar and R. Salehi, J. Nanopart. Res., 2015, 17, 382.

36 A. M. Rasmussen, R. M. Gibson, S. E. Godtfredsen and M. Ottesen, Carlsberg Res. Commun., 1985, 50, 73-82.

37 K. Ius, V. M. Serebrennikov, N. A. Zagustina and A. M. Bezborodov, Prikladnaia Biokhimiia I Mikrobiologiia, 2000, 36, 131.

38 Y. Guo, D. Pan, H. Ding, Z. Wu, Y. Sun and X. Zeng, J. Sci. Food Agric., 2015, 95, 1655-1661.

39 W. Buckel, Methods Enzymol., 1986, 125, 547-558.

40 C. Vogel and J. Pleiss, Proteins: Struct., Funct., Bioinf., 2014, 82, 2523-2537.

41 N. Niedzwiecka, J. Gronczewska and E. F. Skorkowski, Fish Physiol. Biochem., 2017, 43, 351-360.

42 V. A. Marlow, D. Rea, S. Najmudin, M. Wills and V. Fülöp, ACS Chem. Biol., 2013, 8, 2339-2344.

43 F. Fusetti, K. H. Schroter, R. A. Steiner, P. I. van Noort, T. Pijning, H. J. Rozeboom, K. H. Kalk, M. R. Egmond and B. W. Dijkstra, Structure, 2002, 10, 259-268.

44 Z. Fisher, J. A. H. Prada, C. Tu, D. Duda, C. Yoshioka, H. Q. An, L. Govindasamy, D. N. Silverman and R. McKenna, Biochemistry, 2005, 44, 1097-1105. 